\section{FLOATING MAGNETS}

THE publication of my experiments on "Floating Magnets," in the American Journal of Science and in NATURE, was made merely as a claim to this new method of experimenting. I now send you the law of the morphology of their configurations, and show how these experiments illustrate the phenomena of allotropy,

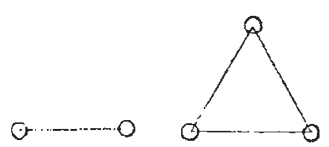

$x / 2$

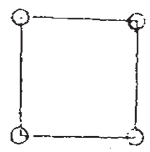

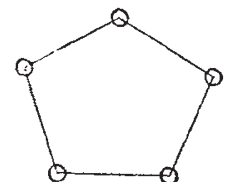

$5<x$ isomerism, expansion or solidification of water, bismuth antimony, \&c., the atomic hypothesis, and the kinetic theory of gases.

The configurations of the floating magnets given in this paper are reduced to half-size. They were obtained as follows:- A cylindrical magnet, 387 millimetres long

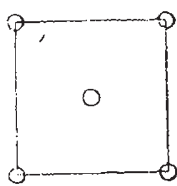

53

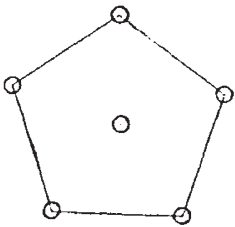

$6 i t$

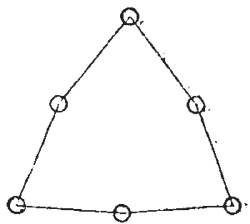

and thirteen millimetres in diameter, was clamped with its lower end sixty millimetres above the plane in which were the ends of the floating magnets.

After each configuration was formed the tips of the needles were dotted with printer's ink, and a flat piece of cardboard was carefully lowered on to the configura-

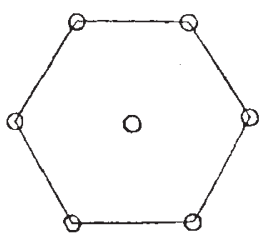

7

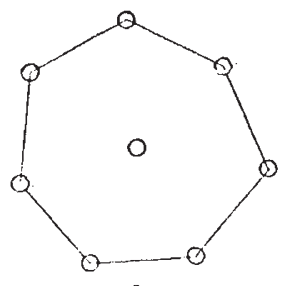

$8 i 2$ tion, which was thus printed on the card. The points formed in this way were placed on drawing-paper, and the imprinted points were pierced with a needle. Thus the centres of the magnets were located, and around these points were drawn the circles of the element of the configurations. The configurations here given are
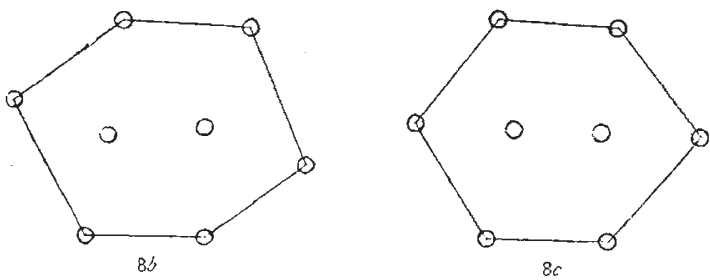

one-half the size of the prints taken from nature, "with all their imperfections on their heads," produced by the unavoidable unequal magnetization of the component needles.

These configurations are numbered from 2 up to $18 b$; the numbers indicating the numbers of floating magnets

in the configurations. Where $a, b$, and $c$ occur under a configuration they show the order of their stability. Thus $5 a$ is more stable than $5 b$ and $6 a$ than $6 b$.

The law of the morphology of these forms is as follows:- They are divided into primaries, secondaries, tertiaries, \&c. The primary configurations are from 2 up to $9 a$.

The secondaries begin with 9 (one might even say with $b$ and $c$ of 8). These secondary configurations have the

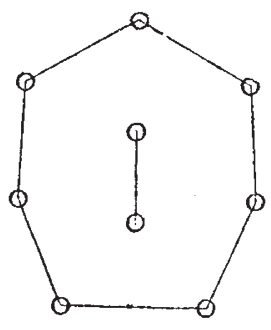

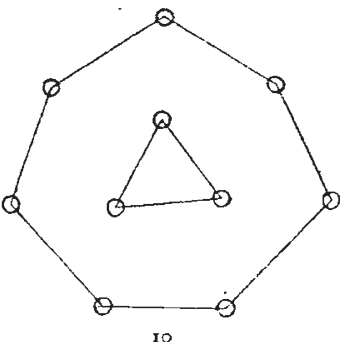

stable primaries for nuclei. Thus configurations 9, IO, II, $12,13,14,15,16,17,18 a$, and $18 b$, have respectively 2 , $3,3,4,4,5$ (flattened), $6 a$ (which is " 5 flattened" expanded to a regular pentagon), 7 , pointed (compressed?) towards a vertex of the hexagon, $7,7,8$.

Nineteen needles form the first configuration of the tertiaries. This is formed of 9 as nucleus, surrounded by ro floating magnets.

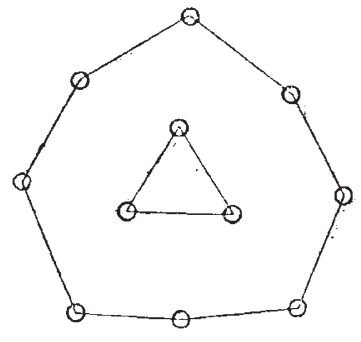

I

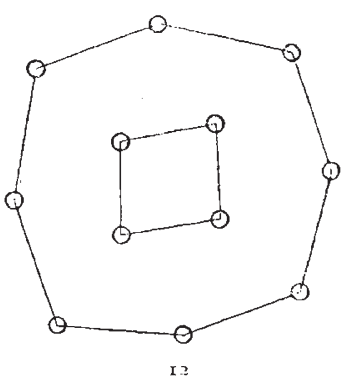

Twenty has 9 for nucleus, with I I circumposed; but this form is unstable, and soon changes into Fig. 20, which has ro magnets for nucleus with 10 circumposed. This is the only instance (except the flattened pentagon, Fig. 14) I have found where a nucleus is changed in form by the action of the circumposed magnets. This nucleus of 20 cannot be formed without the circumposed magnets, as in Fig. 20.

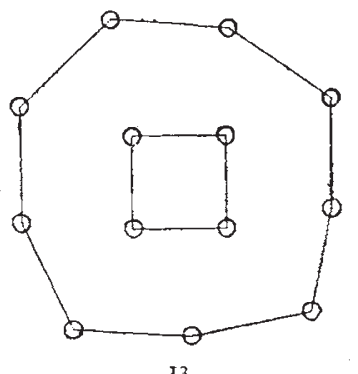

Twenty-two has I I for nucleus, surrounded with il magnets.

Tzenty-three has I I for nucleus, with 12 circumposed needles, arranged parallel to nucleus.

Tzenty-four is formed of I I for nucleus, surrounded with 12 , and one opposite the base of :

Twenty-five is formed of 13 for nucleus, with $12 \mathrm{cir}$ cumposed, and parallel to nucleus. 
Twenty-six is formed of 14 for nucleus, with 12 magnets circumposed.

Twenty-seven is formed of I5 for nucleus, with 13 magnets circumposed.

Twenty-eight is formed of 14 for nucleus with 13 circumposed.

Twenty-nine is formed of 16 for nucleus, with $13 \mathrm{cir}$ cumscribed.

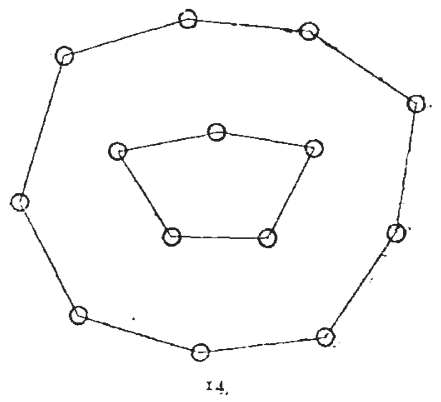

Thirty is formed of 17 for nucleus, with 13 circumscribed.

Thirty-one is formed of 18 for nucleus, with 13 circumscribed.

Thirty-two begins the Quaternary Configurations, for it is formed of I9, with i 3 circumposed magnets.

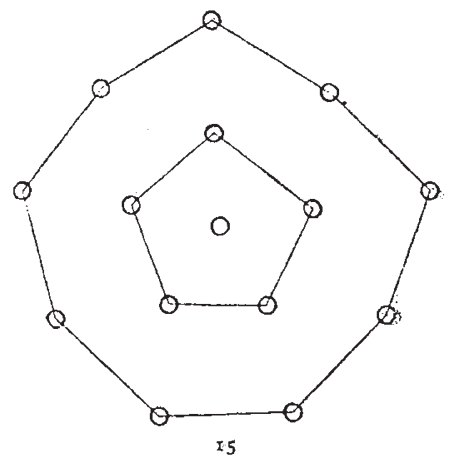

Thirty-three is formed of 20 for nucleus, with $\mathrm{I}_{3}$ magnets circumposed.

Thirty-four is formed of $2 \mathrm{I}$ for nucleus, with $13 \mathrm{mag}$ nets circumposed.

Thirty-five is formed of 22: feet for nucleus, with 13 magnets circumposed.

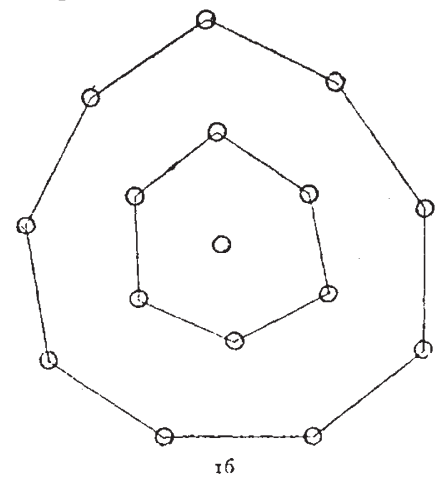

Thirty-six is formed of 23 for nucleus, with 13 magnets circumposed.

Thirty-seven is formed of 24 for nucleus, with 13 circumscribed magnets.

Thirty-eight is formed of 26 with 12 magnets circumscribed.
The expansion of liquids, like ice and antimony, on solidification, allotropy and isomerism, are illustrated by the fact that different configurations formed of the same number of atoms have densities inversely as their areas.

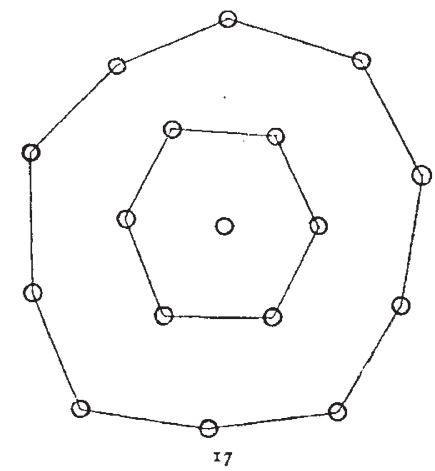

Thus $5 b$ is about $\frac{1}{10}$ th greater in area than $5 a$. So if $5 a$ represent water at $0^{\circ} \mathrm{C}$., $5 b$ may stand for ice at $0^{\circ} \mathrm{C}$.

Similarly in allotropy if $6 a$ stand for graphite, then $6 b$ may stand for diamond, and the three forms of titanic oxide, rutile, brookite, and anatase, and their different densities may be illustrated by configurations $\mathrm{I} a, 8 b, 8 c$.

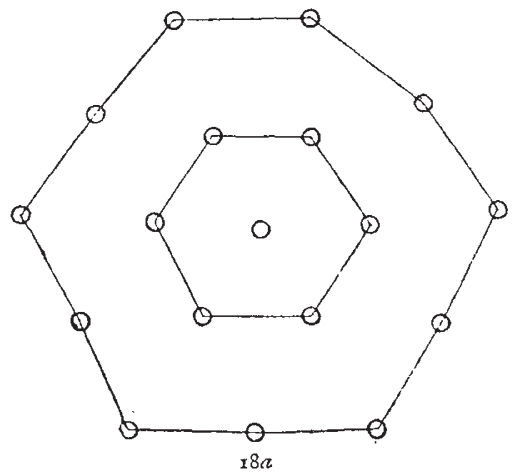

If $6 b$ stand for calcite, then $6 a$ will stand for its isomer anagonite.

The law ruling the density of the configuration is evidently that a central magnet always expands the contours of the configuration. For example, compare Figs. 4 and $5 b, 5 a$ and $6 a, 6 b$ and $6 a, 14$ and 15 .

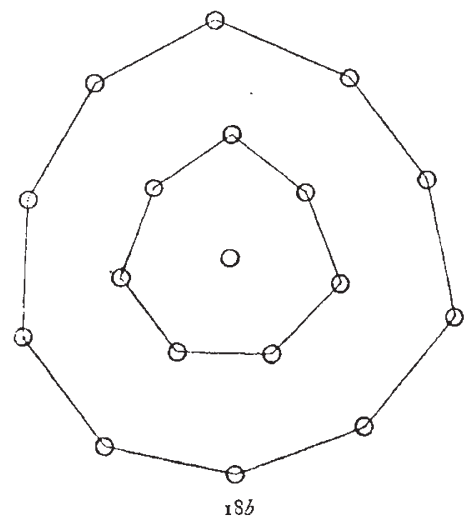

These configurations - at least the stable ones-can be obtained by suspending the magnets by fine silk fibres. I have thus obtained all the stable forms; and the plan proposed to me by my friend Prof. Rood will no doubt give these configurations. He proposed to me to suspend 
gilt pith balls by silk fibres and then electrify them with the same electricity.

If suspended configurations be brought near each other we will cause the vibrations of their component magnets (atoms), and thus we may illustrate the atomic vibrations in molecules. If a suspended configuration be brought in contact with a piece of paper, supported vertically, the interaction of the suspended magnet may force it from the

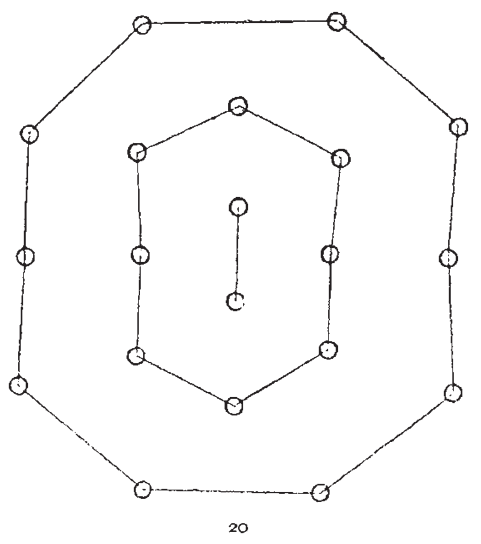

vertical, and cause it to fall, and thus may be illnstrated the molecular pressure of gases.

I will here point out the stable and unstable configurations. $5 a$ is more stable than $5 b$, and $6 a$ is more stable than $6 b$. The latter is sent into $6 a$ on vibrating it. $8 c$ is very unstable (like.. $)$, and goes into $8 b$ on vibration, caused by elevating and lowering the superposed magnet.

A. M. MAYER

\section{P.S. ${ }^{1}$ - As to the configuration}

it is so very un-

stable that I have not reproduced it in these configurations, for it is really too unstable to exist except for $a n$ instant.

The bexagon only exists with a central magnet. Mr. C. S. Pierce and I have had several discussions about the stability of . . I always have maintained that it was impossible to get this form, for a central repellant body was necessary to the tension of the.

which is like a soap bubble without cohesion of contiguous elements. Seven magnets form only. . .

$$
\text { is more stable than }
$$

\section{ON A REMARKABLE FLASH OF LIGHTNING ${ }^{2}$}

( $N$ the evening of August i6 last year (I877) a heavy thunderstorm took place in this vicinity (South port). It was preceded by a fall of the barometer not exceding one-tenth of an inch, the wind at I o'clock P.M. being west, backing gradually until at $90^{\circ}$ clock P.M. it was south. At the time of the storm to which my present observations refer it was south-west, and conse-

Adidressed to Sir $\mathrm{Wm}$. Thomson.

a Papcr by B. St. J. B. Joule, at the Lit. and Phil. Soc., Madchester. quently its direction was nearly parallel with the coast line.

I was standing at the shore-end of Leicester Street, watching the approach of the storm, and observing the progress and direction of the more important flashes, when about $8 o^{\prime}$ clock a vivid flash of lightning fell apparently into the channel (the water being not much above low water mark of a neap tide) about one-sixtb of a mile north of the end of the pier. In about a minute afterwards another fell about one-sixth of a mile north-east of the previous one, and after a similar interval a third stream of electricity descended about another one-sixt of a mile in the same direction. The first and third flashes were of the usual character of forked lightning, but the second presented an appearance which I do not recollect to have witnessed before. From its exit from the clouds to its fall into the sea it seemed composed of small detached fragments which caused it to assume the aspect depicted below.

On the following day, in the course of a conversation respecting the storm of the previous evening, I mentioned the phenomenon to $\mathrm{Mr}$. Thistlethwaite, who informed me that he had been particularly struck by the extraordinary

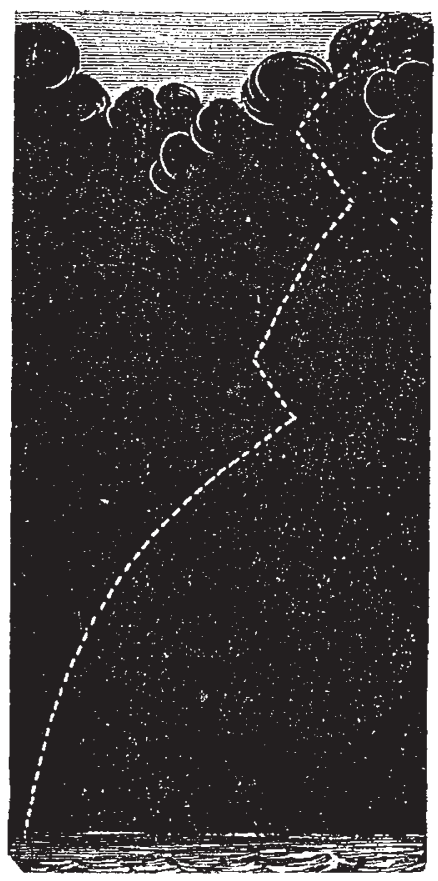

appearance of this singular flash, which he had observed whilst sitting in the "parsonage" (the bouse adjoining the south-west side of the Manchester and Liverpool District Bank), and which to him appeared exactly as I have depicted it. This gentleman could, however, have seen the upper portion of the flash only, as the houses in Lord Street and on the Promenade intervened between his point of observation and the shore.

Heavy rain seemed to follow in the wake of the thircl flash, and came on with a noise like that of a great rush of wind, but as the direction of the storm was nearly coincident with the water-line, inclining but slightly towards the beach, about ten minutes elapsed before the downfall reached the place where I stood.

From the information I afterwards obtained the thunderstorm was subsequently, a few miles to the north-east of Southport, more severe than it was in Southport itself

Southport, March II 